\title{
Polypharmacy: one of the greatest prescribing challenges in general practice
}

Pharmaceutical interventions are addressed by a number of papers in this month's issue of the journal. Drug therapy is the major intervention offered by the NHS to enhance and sustain the health of the population. Medicines cost the NHS in excess of $£ 10$ billion annually, with the total cost and number of prescriptions steadily rising; the majority of prescribing occurs in general practice. Given this investment, together with the shift of chronic disease management to primary care, GPs need to ensure their prescribing is effective in maximising health gains while minimising risks to patients.

\section{THE EVIDENCE}

Polypharmacy is arguably one of the most pressing prescribing issues. There is no formally accepted definition, but it is usually considered as concurrent prescribing of at least four or five drugs. In a German study, over a quarter of older patients in primary care were found to be on five or more medicines, ${ }^{1}$ and a recent Italian study found that over $35 \%$ of older patients were exposed to polypharmacy. ${ }^{2}$ Data from the Swedish Prescribed Drug Register also show a prevalence of over $5 \%$ of patients in their 40 s, and around $12 \%$ of those in their 50 s who are taking multiple medicines. ${ }^{3}$ Rates are increasing in almost all ages, although more so in older people. ${ }^{3}$ And crucially, polypharmacy is associated with undesirable outcomes. ${ }^{4}$

In this issue of the Journal, linked prescription and survey data are used to demonstrate that multiple drug use in older patients is associated with overall worsening physical and psychological health. ${ }^{5}$ Larger studies are required to explore this in further detail, accounting for factors such as drug indication, comorbidities, medication interactions, and duration of treatment. Polypharmacy also has the potential to influence many aspects of safe prescribing, including adverse drug reactions, risk of medication interactions, and adherence. Furthermore, the appropriateness or efficacy of a drug given in combination with many other medications may be unclear. Human error is also likely to be exacerbated due to the complexity of patients' medication regimes. A lack of familiarity with some newer drugs, particularly those initiated in secondary care, may aggravate these issues still further.

Polypharmacy is associated with age, morbidity, and poor self-rated health. ${ }^{6}$ Also, older people are particularly prone to adverse consequences due to agerelated physiological changes altering the pharmacokinetic and pharmacodynamic characteristics of many medicines. ${ }^{7}$ Given that life expectancy is increasing, and multiple morbidity is more common in older patients, the problem of polypharmacy is likely to become worse. Furthermore, our practice of evidencebased and protocol-driven medicine helps to drive the relentless increase in the number of pills a patient is asked to pop; guidelines are usually developed around single conditions and are poorly equipped to inform clinicians how to deal with patients already taking medicines for additional diverse clinical problems.

\section{ADDRESSING POLYPHARMACY}

One way of helping patients to manage multiple medicines is the use of medication compliance aids. In a qualitative study, Lecouturier and colleagues found that such aids are perceived by patients to reduce disruption, improve adherence, and streamline medication taking. ${ }^{8}$ The study also suggests that GPs may be failing to ask patients about these issues, and that problems of communication with respect to polypharmacy occur at the primary-secondary care interface. It should be noted, however, that medication aids do not address the underlying problem of polypharmacy.

Polypharmacy is a difficult issue to address, requiring a rational and structured approach. We need to challenge the current culture of 'ratcheting up' numbers of medications, and to increase awareness of the consequences of polypharmacy. This can be addressed, in part, through continued medical education, and also through clinical guidelines, particularly for common conditions affecting older patients. In addition, tools need to be developed to identify individuals who are likely to be at particular risk from polypharmacy. These may be practicebased or pharmacy-based, and should take into account factors such as age, sex, clinical conditions, or deprivation. ${ }^{6}$ Furthermore, although it may be easier to apply such tools in a retrospective manner, prospective systems would be preferable, especially if they influence prescribing behaviour and prevent polypharmacy.

Polypharmacy is not synonymous with inappropriate prescribing, but the two are nevertheless strongly associated. ${ }^{9}$ Therefore, it would also be valuable to employ validated processes to identify inappropriate prescribing, such as the Screening Tool of Older Person's Prescriptions (STOPP). ${ }^{10}$ The development of prescribing safety indicators for UK general practice, derived from STOPP and other sources, will be published in a forthcoming issue of the BJGP.

\section{MEDICATION REVIEW}

Regular and thorough medication review is an essential intervention for addressing polypharmacy. It is important to assess whether patients are receiving therapeutic benefit from their medicines, whether there is ongoing clinical need, and 
whether potential benefits are outweighed by risks and side-effects. Wherever possible, patients' views should be ascertained; they should be fully involved in decisions about their medicines; the rationale behind any medication changes should be explained; and any concerns should be addressed. Reviews should be specifically arranged, rather than rushed impromptu additions to the end of a 10minute consultation.

Managing patients with polypharmacy can be time consuming, with complex cases requiring careful balancing of competing clinical priorities and conflicting guidelines. Where GPs do not feel they have the time or skills to undertake a thorough medication review, they need to consider alternative approaches, such as employing clinical pharmacists or seeking advice from community geriatricians.

Polypharmacy is a major and growing public health issue. Proactively addressing the problem has significant potential to maximise quality of life for patients, help patients to manage their own medicines, reduce adverse effects, and encourage more rational and efficacious drug use. Further studies are needed to ascertain whether reducing polypharmacy results in better clinical outcomes.

\section{Rupert A Payne,}

Clinical Lecturer in General Practice, General Practice and Primary Care Research Unit, University of Cambridge, Institute of Public Health, Cambridge.

\section{Anthony J Avery,}

Professor of Primary Healthcare, Division of Primary Care, The Medical School, University Hospital, Nottingham.

\section{Provenance}

Commissioned, not externally peer reviewed.

\section{REFERENCES}

1. Junius-Walker U, Theile G, Hummers-Pradier E. Prevalence and predictors of polypharmacy among older primary care patients in Germany. Fam Pract 2007; 24(1): 14-19.

2. Slabaugh SL, Maio V, Templin M, Abouzaid S. Prevalence and risk of polypharmacy among the elderly in an outpatient setting: a retrospective cohort study in the Emilia-Romagna region, Italy. Drugs Aging 2010; 27(12): 1019-1028.

3. Hovstadius B, Hovstadius K, Astrand B, Petersson G. Increasing polypharmacy - an individual-based study of the Swedish population 2005-2008. BMC Clin Pharmacol 2010; 10:16

4. Milton JC, Jackson SHD. Inappropriate polypharmacy: reducing the burden of multiple medication. Clin Med 2007; 7(5): 514-517.

5. Kadam UT. Potential health impacts of multiple drug prescribing for older people: a case-control study. $\mathrm{Br} \mathrm{J}$ Gen Pract 2011; 61(583): 128-130.

6. Moen J, Antonov K, Larsson CA, et al. Factors associated with multiple medication use in different age groups. Ann Pharmacother 2009; 43(12): 1978-1985.

7. Spinewine A, Schmader KE, Barber N, et al. Appropriate prescribing in elderly people: how well can it be measured and optimised. Lancet 2007; 370(9582): 173-184.

8. Lecouturier J, Cunningham W, Campbell D, Copeland
R. Medication compliance aids: a qualitative study of users' views. Br J Gen Pract 2011; 61(583): 93-100.

9. Cahir C, Fahey T, Teeling M, et al. Potentially inappropriate prescribing and cost outcomes for older people: a national population study. Br J Clin Pharmacol 2010; 69(5): 543-552.

10. Gallagher P, Ryan C, Byrne S, et al. STOPP (Screening Tool of Older Person's Prescriptions) and START (Screening Tool to Alert doctors to Right Treatment). Consensus validation. Int J Clin Pharmacol Ther 2008; 46(2): 72-83.

DOI: 10.3399/bjgp11X556146

ADDRESS FOR CORRESPONDENCE

\section{Anthony J Avery}

Division of Primary Care,

The Medical School, University Hospital, Nottingham, NG7 2UH.

Email: tony.avery@nottingham.ac.uk 